[Aus dem hygienischen Institut der Universitït Breslau.]

\title{
Versuche an Meerschweinchen über die Aufnahme inhalierter Tuberkelbazillen in die Lunge.
}

\author{
Von
}

Privatdozent Dr. Bruno Heymann, Leiter der Wutschutr-Abteilung am Institut.

Die Ermittlung der primären Invasionsstätte inhalierter Tuberkelbazillen kann experimentell dadurch versucht werden, daB man an Inhalationstieren die Bazillen etappenweise auf dem Wege verfolgt, welchen sie nuch ihrer Aufnahme rom Nasenrachenraume aus weiterhin nehmen. Für den Nachweis der Bazillen stehen zwei Methoden zu Gebote:

1. die „biologische", - Weiterimpfung der in Betracht kommenden Organe auf Meersehweinchen;

2. die ,mikroskopische", - Untersuchung dieser Organe auf Tuberkelbazillen, bzw. auf durch diese hervorgerufene Gewebsveränderungen.

Nach beiden Richtungen liegt bereits einiges Naterial vor: Max Wolff ${ }^{1}$ hat in einer Diskussionsbemerkung zu Westenhoeffers Vortrag „Über die Wege der tuberkulösen Infektion im kindlichen Körper" berichtet, $\mathrm{da} B$ er Tiere unmittelbar nach der Inhalation tuberkelbazillenhaltigen Materials getötet, mit den Lungen dieser Tiere andere infiziert habe, und dab sich die Lungen bei der einen Versuchsreihe in 75 Prozent, bei einer anderen in 100 Prozent tuberkelhaltig erwiesen haben. Ausführlichere Mitteilungen über diese Versuche sind bisher nicht erschienen. Wolff zieht aus ihnen den SchluB, daB, die primäre direkte Infektion des Respirationsapparates auf dem Wege der Einatmung als die hauptsächlichste Quelle der Infektion angesehen werden muB".

${ }^{1}$ Max Wolff, Berliner klin. Wochenschr. 1904. S. 256. 
Weleminsky ${ }^{1}$ lieb ein Kaninchen zusammen mit zwei Meerschweinchen $1 / 2$ Stunde lang einen Tuberkelbazillenspray atmen, tôtete es unmittelbar danach und brachte ein Stück Lunge einem Meerschweinchen unter die Haut. Dieses blieb völlig gesund, nach Weleminsky ,ein Beweis, daB die Bazillen überhaupt nicht bis in die Lunge gekommen waren". Ein solcher SchluB ist indessen nicht statthaft. Ganz abgesehen davon, daB das Ergebnis eines einzigen Versuchs stets nur mit äußerster Zurückhaltung verwertbar ist, erfordert der vorliegende Versuch noch eine ganz besonders vorsichtige Beurteilung: Zunächst ist die Technik der Weiterimpfung eine ungeeignete. Wie die unten besprochenen Versuche von Bartel und Neumann ${ }^{2}$ beweisen, kann die subkutane Verimpfung ganzer Gewebsstücke selbst bei tuberkelbazillen reichen Material versagen. Nun sind aber alle weiteren Angaben über die Versuchsbedingungen so wenig genau, daß gar keine Möglichkeit besteht, die Menge der von dem Kaninchen etwa inhalierten Bazillen auch nur annähernd abzuschätzen, sondern man ist in dieser wichtigen Frage auf Vermutungen angewiesen. Da Weleminsky an anderer Stelle ${ }^{3}$ ausdrücklich darauf hingewiesen hat, daB bei Inhalationsexperimenten zur besseren Nachahmung der natürlichen Verhältnisse geringe Dosen von Infektionsmaterial besonders geeignet seien, so läßt sich annehmen, daß auch bei diesem Versuch nur relativ wenige Bazillen versprayt, und damit die Aussichten für ihren Nachweis noch vermindert wurden. Vielleicht hat ferner der Mangel einer Fixierung der Versuchstiere während. der Inhalation in diesem Sinne erheblich mitgewirkt. Wenn man den Kopf der Tiere nicht irgendwie, wenn auch in schonendster Weise und in möglichst ungezwungener Stellung, fixiert, so kommt es leicht dazu, daB durch enges Aneinanderschmiegen der Tiere, Verkriechen des Kopfes in eine dem Spray abgewandte Ecke und dgl. die Aufnahme des Spraynebels erschwert wird. Dieses Mroment kann bei dem Kaninchen sehr wohl eine Rolle gespielt haben, wenn auch die zur Kontrolle dienenden Meerschweinchen erkrankten. Vor allem aber steht das Ergebnis dieses Versuchs zu den positiven Resultaten sehr vieler analoger Experimente anderer Forscher durchaus im Widerspruch. Trotz alledem hat Wele minsky aus seinem einen negativen Ergebnis weitgehende, allgemeine

\footnotetext{
1 Weleminsky, Zur Pathogenese der Tuberkulose. Autoreferat. Ans dem Sitzungsberichte des Vereins deatscher Ärzte. in Prag, 3. März 1905. Prager med. Wochenschrift. 1905. XXX.

${ }^{2}$ Bartel und Neumann, Über experimentelle Inhalationstuberkulose bein Meersehweinchen. Wiener klin. Wochenschrift. 1906. Bd. XIX.

${ }^{3}$ Weleminsky, Zur Pathogenese der Lungentuberkulose. Berliner klin. Wochenschrift. 1905. Nr. 31 u. 32.
} 
Folgerungen über die Infektionswege der inhalierten Bazillen, selbst auch für den Menschen, abgeleitet, und diesen Folgerungen haben sich, offenbar ohne Nachprüfung und ohne Würdigung der unzulänglichen Grundlage des Versuchs, merkwürdigerweise zahlreiche andere Autoren angeschlossen.

Ková s $^{1}$ ließ junge Meerschweinchen in zwei Versuchsreihen „zweierlei Infektionsstoff in einer geeigneten Glocke verstäubt einatmen; bei der ersten Gruppe wurde mehrere Tage hindurch im Vakuum bei Zimmertemperatur getrocknetes und so verriebenes und zerstäubtes Sputum 3 Minuten lang inhaliert, und zwar ist insgesamt $10^{\mathrm{cm}}$ Sputum getrocknet, verrieben und durch 8 Tiere eingeatmet worden; die zweite Gruppe hat unter denselben Bedingungen mit Zigarrenasche vermengte Tuberkelbazillen 2 Minuten lang eingeatmet, wobei wieder die Asche einer Zigarre mit $1 \mathrm{cg}$ virulenten Kulturmaterials und etwas pulverisiertem Sputum gut vermengt verwendet wurde. - Von beiden Gruppen wurden nach der Inhalation je zwei Tiere getötet, um den Respirationstrakt auf die Gegenwart ron hineingelangten Keimen zu prüfen. - Diese Untersuchung der Lungen wurde mittels Verfertigung mehrerer Quetschpräparate und Verimpfung von Lungenstücken in die Bauchböhle durchgeführt."

Angesichts der vielfach irrtümlichen Verwertung der Kovácsschen Versuchsergebnisse habe ich die - hier ausschlieBlich interessierenden Inhalationsversuche nach den Originalprotokollen in Tabelle I kurz zusummengestellt. Am bedeutsamsten ist hiernach der direkte Nachweis von Tuberkelbazillen (einmal) im Kehlkopf und (zweimal) in der Lunge sofort nach der Inhalation. Bemerkenswert ist ferner die Beschränkung der pathologischen Befunde aussehlieBlich auf die Submaxillar-, Cervical-, Tracheal-, Bronchialdrüsen and die Lungen, während am Intestinaltraktus und seinen regionären Drüsen Abnormes nicht gefunden wurde. Allerdings hat sich Kovács offenbar nur mit ihrer makrostiopischen Besichtigung begnügt. Hier wie auch bei der Cntersuchung der in der Tabelle aufgeführten Organe wäre eine ausgedehntere Anwendung der Verimpfung auf andere Tiere erwünscht gewesen. Nur in zwei Fällen, und zwar mit Lungen, ist von ihr Gebrauch gemacht worden; beide Male mit negativem Ergebnis. Dieser, besonders bei dem Tier I, 1 (mit positivem Bazillenbefund im Kehlkopf) auffällige Mißerfolg erklärt sich indes unschwer aus dem wahrscheinlich nicht recht geeigneten Inhalations-

\footnotetext{
1 Kováes, Was ergibt sich in bezug anf die Pathogenese der Lungentuberkulose nach Bestimmung der Infektionswege bei Fütterang und Inhalationsversuchen? Zieglers Beiträge. 1906. Bd. XL. S. 281.
} 
Adfnahme inhalimeter Tuberkelbaziluen in DIe Lunge. 493

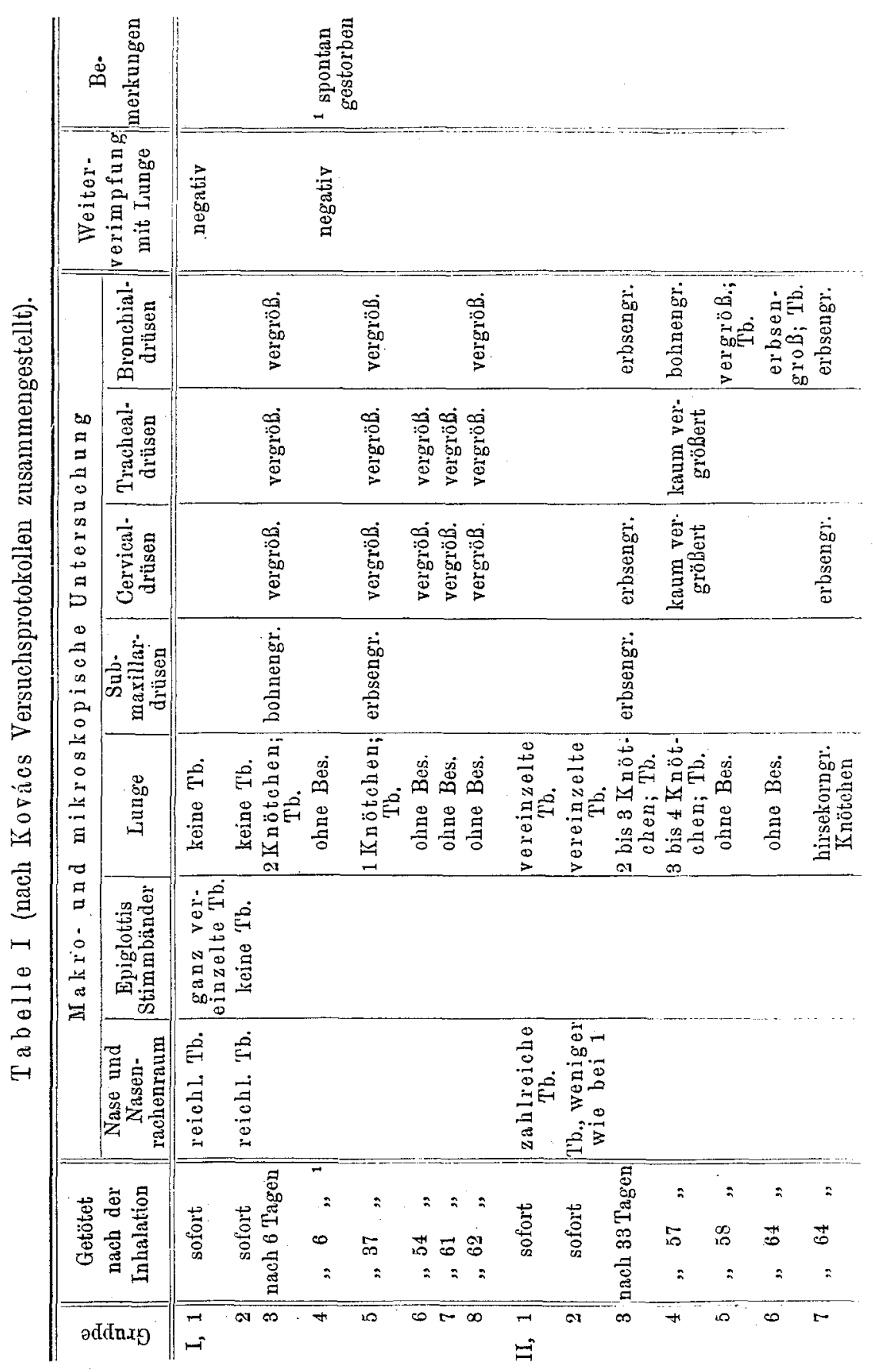


material, das infolge der tagelangen scharfen Trocknung wobl nur noch teilweise virulent und auBerdem vielleicht zu grobkörnig war; es ist ja eine längst bekannte Tatsache, daB mit trockenem, staubförmigem Material Inhalationsexperimente mit Tuberkelbazillen sehr häufig mißlingen (vgl. die unten abgedruckte Arbeit von Koehlisch). Nur so erklären sich auch die spärichen positiven Ausschläge überhaupt: Von sechs überlebenden Tieren erweisen sich nur zwei infiziert. Besser war offenbar das Verstäubungsmaterial bei der zweiten Versuchsreihe: Hier sind von fünf überlebenden Tieren vier sicher infiziert, das fünfte hat stark geschwollene Cervical- und Bronchialdrüsen, sowie in der lunge Knötchen, über deren Natur allerdings weitere Angaben fehlen. Doch sind auch bei diesen Tieren selbst 2 Monate nach der Inhalation die Veränderungen relativ gering. Korács kommt dadurch, daB er die Erschwerung der Versuchsbedingungen nicht genügend würdigt, zu einer Unterschätzung des ,direkten Bazillenimports mit dem Inhalationsstrom" gegenüber der „lymphohämatogenen" I ungeninfektion vom Darm aus, welche ihm bei Verfütterung groBer Dosen Tuberkelbazillen-Reinkultur mebrfach gelang. Er hätte sicher ganz andere Eindrücke aus seinen Versuchsreihen gewonnen, wenn er zur Inhalation einen Spray feiner Tröpfchen benutzt hätte, oder einen künstlich aufgewirbelten, ausgewählten Stanb, wie ihn Koehlisch (siehe unten) benutzte.

Bartel und Neumann ${ }^{1}$ setzten alte und. junge Meerschweinehen in einem besouderen Inhalationsapparat einem Spraynebel von Tuberkelbazillen-Reinkulturaufschwemmung verschieden starker Konzentration ( 1 ms Kultur Menschentuberkelbazillen pro Kubikzentimeter in 1/8, 1/10- und $1 / 18$ prozentiger Verdünnung) 2 bis 5 Minuten lang aus, töteten die Tiere verschieden lange nach der Inhalation und untersuchten die in der Tabelle II aufgeführten Organe makro- und mikruskopisch, sowie durch subkutane Weiterimpfung von Organstücken in toto. Für die Lungen erwies sich letzteres Vorgehen als unanwendbar; die so geimpften Tiere gingen in kurzem an schwerem Hautemphysem usw. zugrunde. Die Verfusser haben daher weiterhin die Lungenstücke ,mit Bouillon oder destilliertem Wasser verrieben, die Emulsion filtriert und das Filtrat verimpft".

Unter Weglassung der makro- und milkroskopischen Befunde, welche nur einmal [bei Reihe I, 5 in der angegebenen Weise] von dem Impfergebnis abweichen, habe ich wiederum aus den Originalprotokollen die Resultate der Weiterimpfungen in Tabelle II zusammengestellt. Es zeigt sich, daB die Lungen von 20 Inhalationstieren nur neunmal die geimpften

\footnotetext{
1 Bartel und Neumann, Über experimentelle Inhalationstuberkulose beim Meerschweinchen. Wiener klin. Wochenschrift. 1906. Bd. XIX. Nr. 7 u. 8.
} 
Aufnahme inhalierter Tuberkiblbazillen in die Iunge. 495

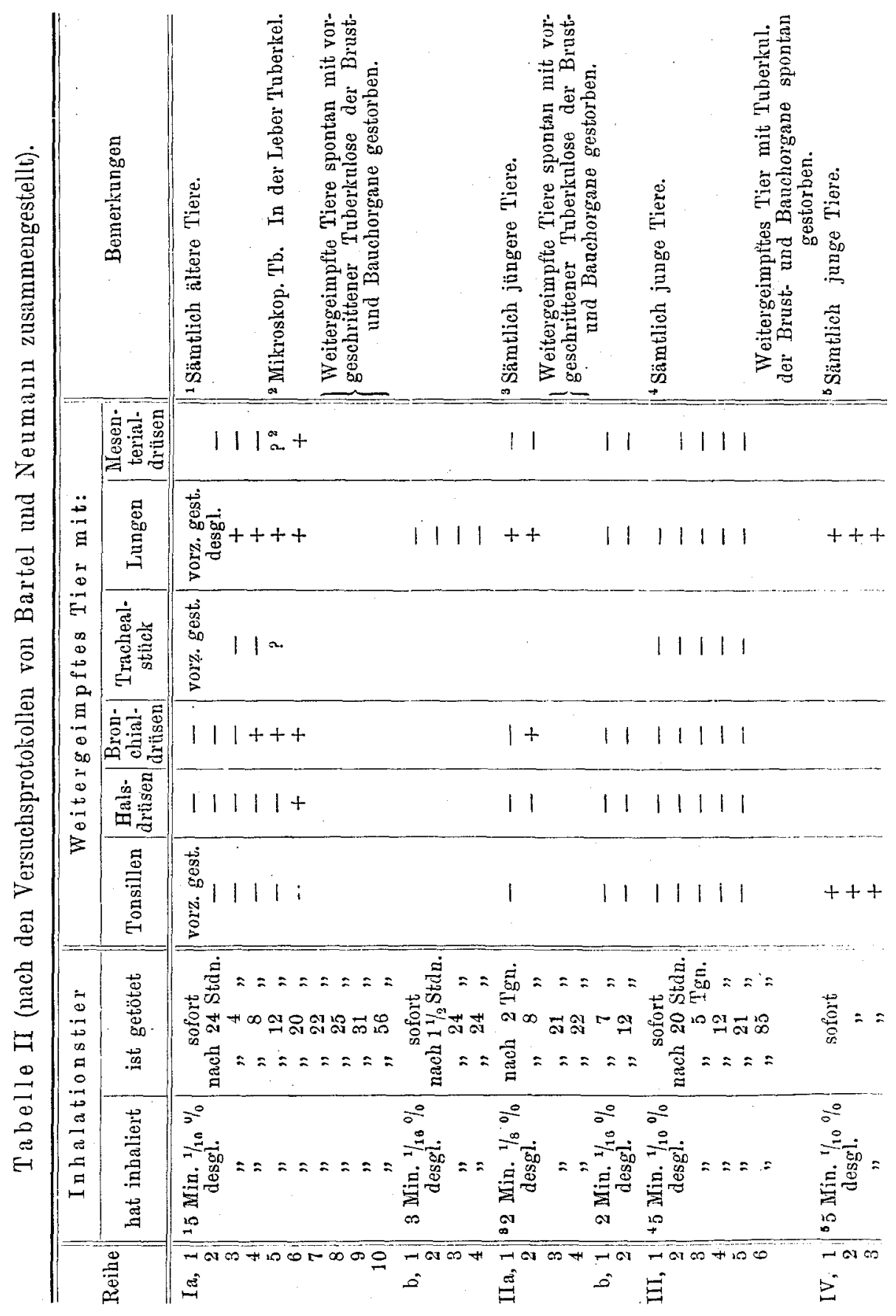


Tiere infiziert haben, und zwar, mit Ausnahme der Versuchsreihe IV, frühestens erst 2 Tage nach der Inhalation. Als Ursache für diese auffälligen Resultate haben Bartel und Neumann selbst ungenügende Zerkleinerung des Lungengewebes, zu starke Verdünnung und die Filtration des Impfmaterials erkannt. Als sie bei der Reihe IV die Iungen, ,mit etwas Quarzsand und Wasser in toto fein zerrieben und die ganze Emulsion ohne vorheriges Filtrieren je einem Meerschweinchen subkutan injizierten", erwiesen sich die Lungen sofort nach der Inhalation säm tlich tuberkelbazillenhaltig. Der Einwand ungeeignater Technik trifft aber auch alle übrigen Weiterimpfungen der ersten drei Reihen; denn auch für sie stellt die Reihe IV einen ausschlaggebenden Kontrollversuch dar: In letzterer ergeben die Tonsillen nach gleicher Vorbereitung wie die Lungen durchwegs positive, bei den drei anderen Reiben durchwegs negative Resultate. Bei den Versuchsreihen $\mathrm{Ib}$ und II b dürfte neben diesen technischen Mängeln wohl noch eine allzu kleine Inhalationsdosis für die negativen Ergebnisse verantwortlich zu machen sein. Unter diesen Umständen fällt es schwer, aus den Versuchsreihen weitergehende Schlüsse abzuleiten, wenn man nicht gerade geneigt ist, den Autoren auf das unsichere Gebiet der „lymphoid hyperplastischen Drüsenveränderungen mit negativem Impfversuch" zu folgen. Beachtenswert ist immerhin, daß die Bazillen stets zuerst in den Lungen, dann, nach 8 Tagen, in den Bronchialdrüsen und erst nach 12 Tagen auch in den Mesenterialdrüsen nachzuweisen waren.

Ein sicheres Urteil darüber, ob inhalierte Tuberkelbazillen wesentlich auf direktem Wege die Iungen erreichen und infizieren oder erst nach der Passage durch die lymphatischen Organe des Rachens und seiner Adnexe oder des Darmes, läßt sich demnach auf Grund der rorliegenden Versuche nicht fällen. - Wohl können wir uns aus analogen Versuchen mit feinsten Körperchen und mit anderen Bakterien eine vorläufige Ansicht bilden, und diese lautet zweifellos zugunsten des direkten Imports. Arnold ${ }^{1}$ hat in seinen bekannten Untersuchungen den Beweis geführt, daß feinste Stäubchen in die Tiefe des Lungenparenchyms inspieriert werden; verschiedenste Autoren haben nach ihm diese Ergebnisse bestätigt, in neuester Zeit die zahlreichen in Ballins vorstehender Arbeit aufgeführten Forscher. Ballin konnte bei seinen Inhalationsversuchen mit Schimmelpilzsporen auch durch mikroskopische Untersuchung den Weg der Pilzsporen verfolgen; er fand sie ausnahmslos in reichlicher Menge in den Alveolen und bereits nach kurzer Frist im interstitiellen Gewebe. - Daß ebenso auch feinste Tröpfehen beim Atmen direkt bis

${ }^{1}$ Arnold, Untersuchungen üher Staubinhalation u. Staubmetastase. Leipzig 1885. 
in die Alveolen gelangen, haben Versuche von von Schrötter ${ }^{1}$ jun. und Heryng ${ }^{2}$ gezeigt, welche gefärbte Flüssigkeiten versprayten. Für mit Bakterien beladene Tröpfchen ist von Buchner, Hildebrandt ${ }^{4}$, Wyssokowitsch ${ }^{5}$, Nenninger ${ }^{6}, \mathrm{Paul}^{7}$, Hartil und Herrmann ${ }^{8}$ durchaus das gleiche Verhalten festgestellt worden. Die von Paul eingehend erörterten und nachgeprüften theoretischen Bedenken Saengers ${ }^{9}$ sowie Buttersacks ${ }^{10}$ werden durch die Ergebnisse hinfällig. Es ist in der Tat kaum zu verstehen, wie nach dem positiven Ausfall so unzähliger Versuche mit analogen Elementen manche Autoren nun gerade für Tuberkelbazillen doch noch die Möglichkeit des direkten Weges in die Lungen in Abrede stellen oder nur ,unter durchaus unnatürlichen Bedingungen" zugeben. Da jedoch dieser Einwand eine endgültige Widerlegung bislang nicht erfahren hat, da namentlieh die quantitative Abstufung der Infelitionsdosis und das Vermeiden reichlich vorhandener Versuchsfehler in den bisherigen Experimenten nicht genügend berücksichtigt war, habe ich versucht, die bestehende Lücke auszufüllen, und berichte im folgenden über einige speziell mit dem Tuberkelbazillus angestellte Versuchsreihen, bei welcben ich mich zum Nachweis der Bazillen teils der biologischen, teils der mikroskopischen Methode bedient habe.

\section{Versuche mit biologischer Prüfung.}

$\mathrm{Zu}$ den Versuchen wurde der von Finde ${ }^{11}$ beschriebene Inhalationsapparat benutzt, in welchem die Versuchstiere (Meersehweinchen von 300 bis $500 \mathrm{grm}$ Gewicht) ohne jede Fesselung in durchaus natürlicher Weise einem feinsten, aus einer Tuberkelbazillenaufschwemmung entwickelten,

1 v. Schrötter jun. Nach freundl., durch Tafeln belegter Mitteilung nicht veröffentlichter Vorversuche zu der gemeinsam mit Kapralik verfaBten Arbeit über Tuberkulininhalationen. Wiener klin. Wochenschrift. 1904. Nr. 21.

${ }^{2}$ Heryng, Untersuchungs- und Behandlungsmethoden der Kehlkopfkrankheiten. Berlin 1905.

s Buchner, Archiv für Hygiene. 1888. Bd. VIII.

4 Hildebrandt, Beiträge zur pathol. Anatomie. Bd. II.

5 Wyssokowitsch, Mitteilungen aus Dr. Brehmers Heilanstalt in Görbersdorf. 1889.

${ }^{8}$ Nenninger, Diese Zeitschrift. 1901. Bd. XXXVIII.

7 Paul, Ebenda. 1902. Bd. XL.

${ }^{8}$ Hartl and Herrmann, Wiener klin. Wochenschrift. 1905. Nr. 30.

- Saenger, Münchener med. Wochenschrift. 1901. Nr. 21. - Virchows Archiv. Bd. CLXXIX.

10 Buttersack, Zeitschrift für klin. Medizin. Bd. XXXIX.

11 Findel, Vergleichende Untersuchungen über Inhalations- und Fütterungstuberkulose. Diese Zeitschrift. 1907. Bd. LVII.

Zeitschr. f. Hygiene. LX. 
Spraynebel ausgesetzt wurden und dabei eine quantitativ berechenbare Menge Bazillen einatmeten. Die Aufschwemmung wurde stets frisch mit Menschentuberkelbazillen in einer Konzentration ron 0.5 oder $0.1 \mathrm{mg}$ Kultur pro Kubikzentimeter destillierten Wassers hergestellt und hierbei auf homogene Verteilung der Bazillen peinlichst geachtet. Die Inhalationsdauer belief sich auf 5 Minuten (in der 2. Versuchsreihe), auf 10 (in der 1.) und auf 50 Minuten (in der 3. Versuchsreihe); die Zeiten und Konzentration entsprachen nach der Findelschen Rechnung der Aufnahme von etwa 10000 Bazillen in der ersten Reihe, von $100000 \mathrm{Ba}-$ zillen in der zweiten und von 1 Million in der dritten. - Nach beendeter Inhalation wurden die Tiere herausgenommen und je eins nach 1 Stunde, nach $12,24,3 \times 24$ und $6 \times 24$ Stunden durch Nackenschlag getötet. Nach Befeuchtung des ganzen Felles mit 5 Promille Sublimatlösung und seiner vorsichtigen Entfernung von der Brust- und Bauchfläche erfolgte sodann mit immer gewechselten, sterilen Instrumenten die Eröffnung der Brusthöhle und die Herausnahme der Brustorgane im ganzen. Nun wurden von den Liungen zunächst die äuBersten Zipfel der Unterlappen (,Lungenzipfel“), hierauf, unter Umgehung der Partien an der Lungenwurzel, noch gröBere Stücke von den einzelnen Lappen ("Lungenrest") abgetragen, schließlich die Bronchialdrüsen vorsichtig von der Trachea abpräpariert, und diese drei Materialien gesondert in sterilen Porzellanmörsern unter Zusatz von steriler Kochsalzlösung so fein zerrieben, daB selbst vou dem Lungenrest fast die gesamte Masse mittels Pravazspritze mit weiter Kanüle Nfeerschweinchen injiziert werden konnte. Die Tiere vertrugen die Injektion sehr gut; kein einziges von ihnen ist kurz nach der Injektion oder aus anderer Ursache vorzeitig zugrunde gegangen. Die Obdnktionsbefunde sowie die Versuchsbedingungen im einzelnen ergeben sich aus den nachfolgenden Protokollen und Tabellen.

\section{Versuchsreihe I.}

Fünf Tiere inhalieren in je 10 Minuten langer Sitzung je 10000 Tuberkelbazillen, werden nach den oben genannten Zeiten getötet und ihre Brustorgane in der beschriebenen Weise auf Meerschweinchen weitergeimpft. Die Termine ihres spontanen Todes bzw. ihrer Tötung sowie die Sektionsergebnisse sind in Tabelle III zusammengestellt. Aus derselben geht herror, daB die Lungen selbst in den periphersten Teilen bereits 1 Stunde nach der Inhalation und ebenso zu allen späteren Terminen Tuberkelbazillen enthalten, während sie in den Bronchialdrüsen erst nach 3 Tagen nachzuweisen waren. Sehr auffällig ist es, daß nach 6 Tagen die Bropchialdrüsen wiederum ein negatives Impfergebnis haben. 


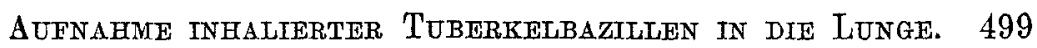

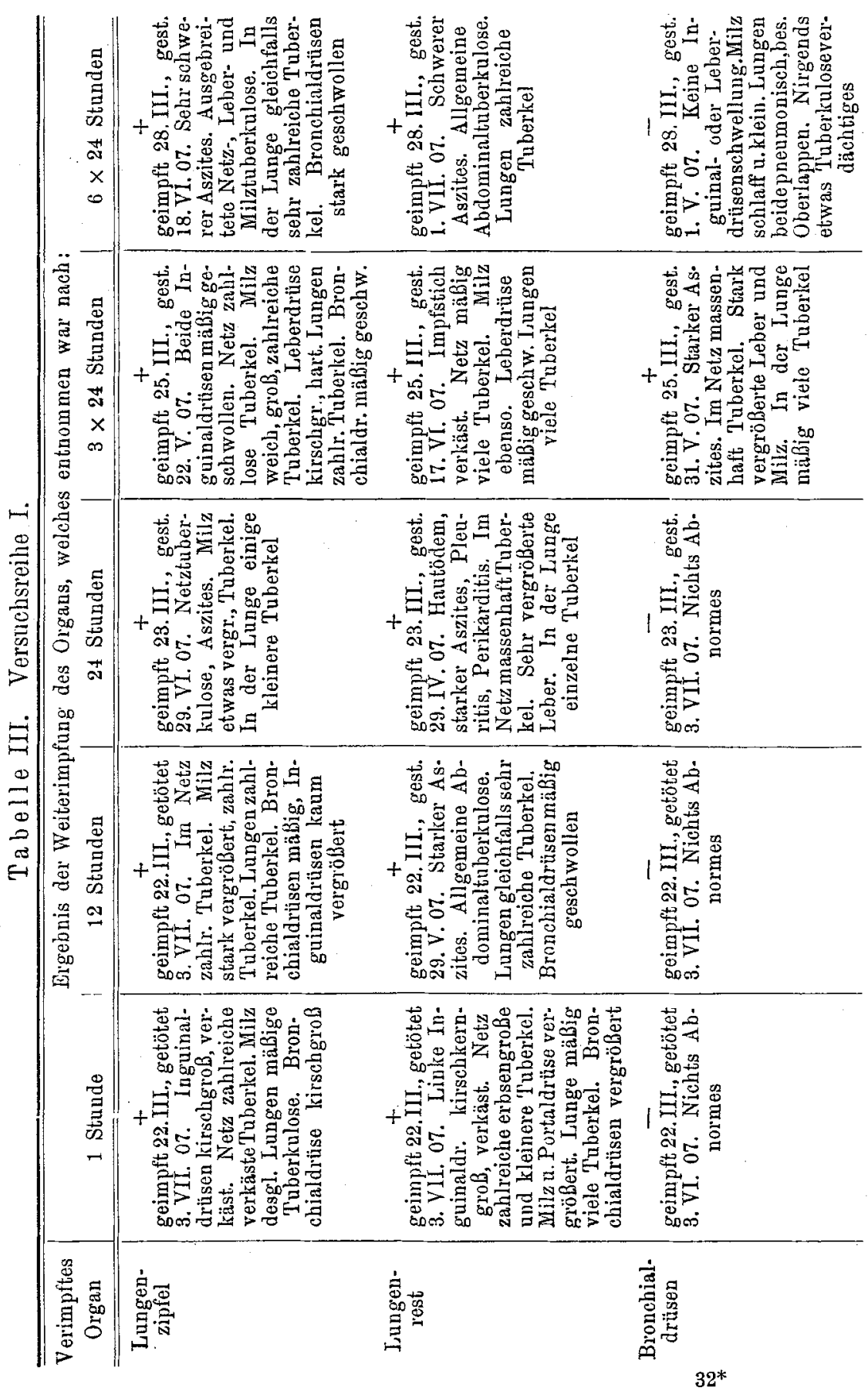




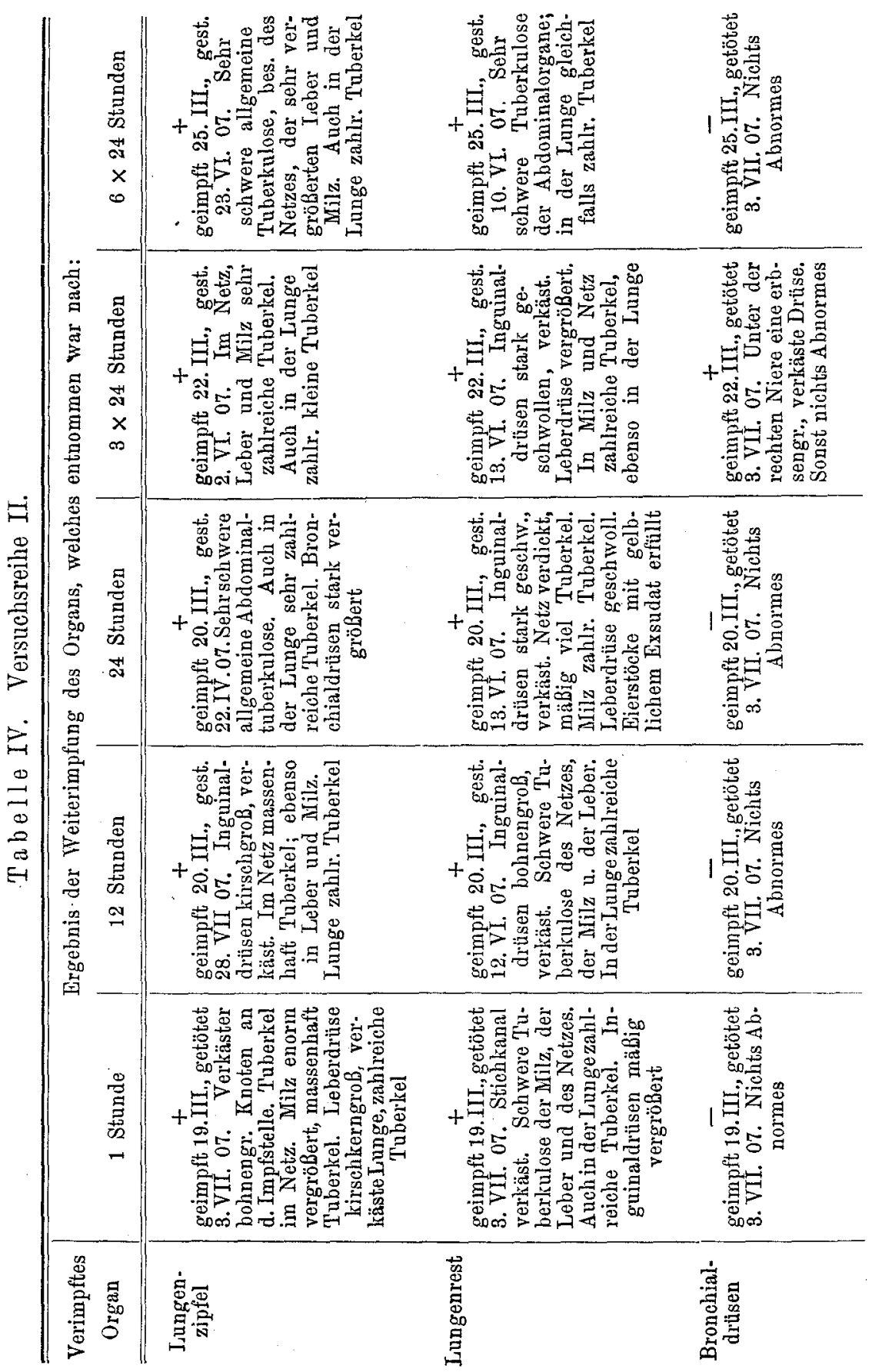




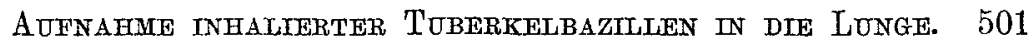

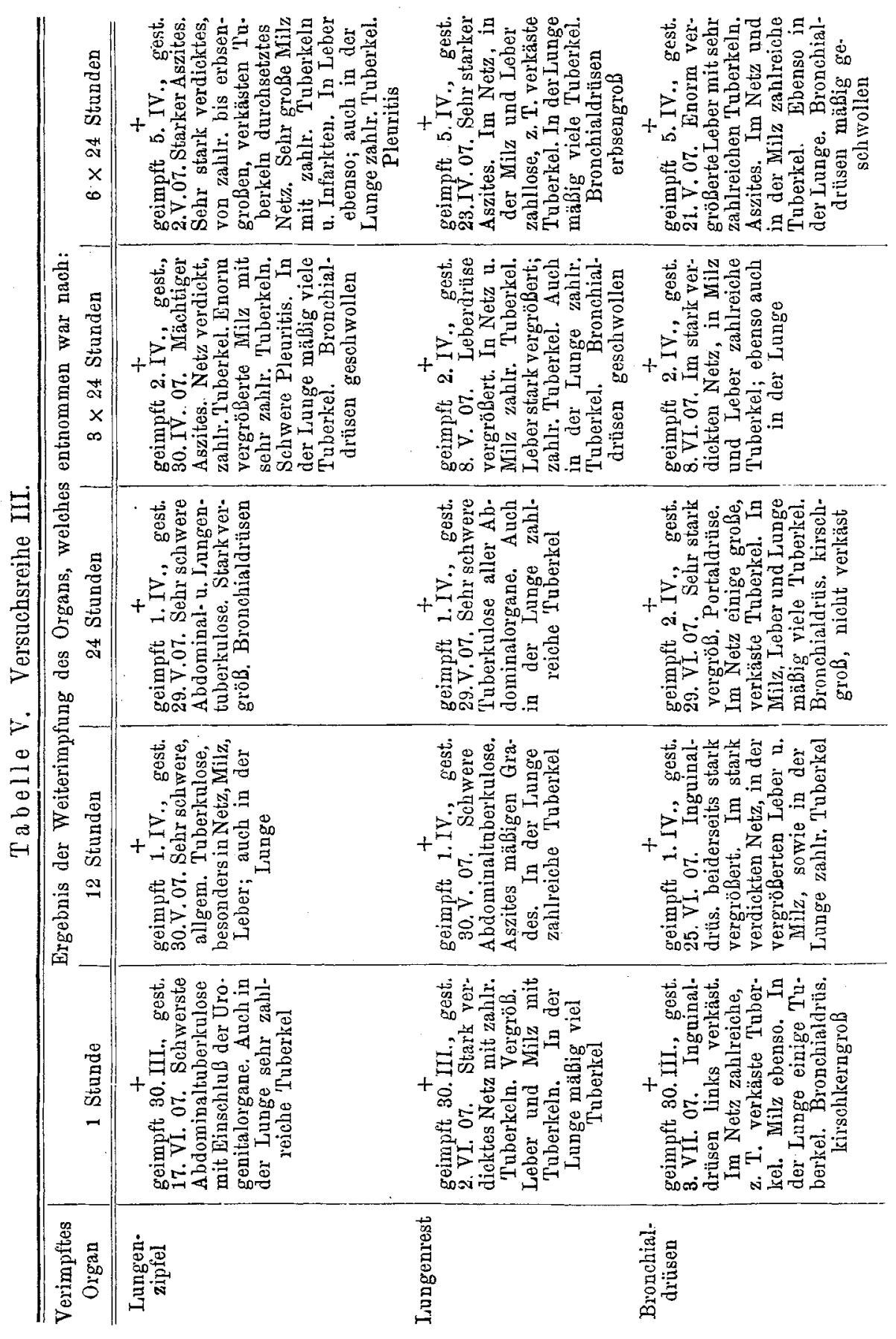




\section{Versuchsreibe II.}

Fünf Tiere inhalieren in je 5 Minuten langer Sitzung je 100000 Tuberkelbazillen. Tötung und weitere Verarbeitung wie in Versuchsreihe I. Die Obduktionsbefunde bei den Impftieren enthält Tabelle IV. Dieselbe stimmt mit Tabelle III vollständig überein. Auch hier erweist sich die Lunge bereits kurz nach der Inhalation und später in allen ihren Teilen infektiös, die Bronchialdrüsen erst nach 3 Tagen. Auch hier haben letztere 6 Tage nach der Inhalation wiederum keinen Impfeffekt.

\section{Versuchsreihe III.}

Fünf Tiere inhalieren in je 50 Minuten langer Sitzung je 1000000 Tuberkelbazillen. Ihre Tötung und weitere Verarbeitung erfolgt in gleicher Weise wie in Reihe I und II. Die Ergebnisse der Weiterimpfungen zeigt Tabelle V. Sie weichen insofern ron denen der beiden ersten Reihen ab, als nun auch die Bronchialdrüsen kurz nach der Inhalation virulente Tuberkelbazillen beherbergen und durch alle geprüften Zeitintervalle hindurch behalten.

Tabelle VI. Gesamtergebnisse.

\begin{tabular}{|c|c|c|c|c|c|c|}
\hline \multirow{2}{*}{$\begin{array}{c}\text { Inhalierte } \\
\text { Bazillen- } \\
\text { menge }\end{array}$} & \multirow{2}{*}{$\begin{array}{l}\text { Verimpites } \\
\text { Organ }\end{array}$} & \multicolumn{5}{|c|}{$\begin{array}{l}\text { Ergebnis der Weiterimpfung des Urgans, } \\
\text { welches entnommen war nach: }\end{array}$} \\
\hline & & 1 Stunde & 12 std. & 24 Std. & $3 \times 24$ Sta. & $6 \times 24$ Std. \\
\hline \multirow[t]{3}{*}{10000} & Lungenzipfel & + & + & + & + & + \\
\hline & Langenrest & + & + & + & + & + \\
\hline & Bronchialdrüsen & - & - & - & + & - \\
\hline \multirow[t]{3}{*}{100000} & Lungenzipfel & + & + & + & + & + \\
\hline & Lungenrest & + & + & + & + & + \\
\hline & Bronchialdrüsen & - & - & - & + & - \\
\hline \multirow[t]{3}{*}{1000000} & Lungenzipfel & + & + & + & + & + \\
\hline & Lungenrest & + & + & + & + & + \\
\hline & Bronchialdrüsen & + & + & + & + & + \\
\hline
\end{tabular}

Behufs besserer Übersicht über alle Reihen habe ich die Gesamtergebnisse nochmals in der Tabelle VI vereinigt. Aus ihr sind folgende zusammenfassende Schlüsse abzuleiten:

1. In den Lungen mittelgroBer Meersehweinchen sind 1 Stunde und später nach der Inhalation eines tuberkelbazillenhaltigen Spraynebels selbst bei mittleren Dosen (10000 Bazillen) stets Tuberkelbazillen nachzuweisen und zwar auch in den periphersten Partien der Lungenbasis. 
2. In den Bronchialdrüsen sind bei mittleren Dosen (10000 and 100000 Bazillen) erst 3 Tage nach der Inhalation Tuberkelbazillen nachzuweisen, nach 6 Tagen wieder nicht mehr. Ob sie inzwischen vernichtet oder weitergewandert sind, ist nach den vorliegenden Versuchen nicht zu entscheiden.

3. Dagegen sind bei bohen Dosen ( 1000000 Bazillen) auch in den Bronchialdrüsen bereits 1. Stunde nach der Inhalation Tuberkelbazillen nachzuweisen und verschwinden nicht mehr aus ihnen. Ob und wie schnell vielleicht ein Teil von ihnen gleichzeitig nach anderen Organen gelangt, müssen weitere Versuche lehren.

\section{Mikroskopischer Nachweis.}

Die Erforschung der primären Ansiedlungsstätte inhalierter Tuberkelbazillen durch mikroskopische Untersuchung der in Betracht kommenden Organe begegnet erheblichen Schwierigkeiten. Am natürlichsten erscheint es, die Bazillen selbst in gewissen Zeitabständen nach der Inhalation aufzusuchen und so ihren anfänglichen Sitz und ihr weiteres Schicksal zu verfolgen. Allein dieser nächstliegende $W_{e g}$ hat bisher zu keinem befriedigenden Ziele geführt: Der Bazillennachweis gelang entweder erst in sehr vorgeschrittenen Stadien der Erkrankung, so dab in dem hochgradig veränderten Gewebe weder die ursprünglich eingebrachten Keime noch ihr erster Angriffspunkt feststellbar waren, oder aber er glückte in früheren Stadien, war dann jedoch durch ganz unnatürliche Versuchsbedingungen, insbesondere durch Einführung massenhaften Infektionsmaterials, erzielt und gestattete keinen RüekschluB auf die Vorgänge unter natürlichen Verhältnissen. Will man aber angesichts dieser Mißerfolge auf den Nachweis der Erreger ganz verzichten und sich lediglich mit der Feststellung der durch sie ausgelösten pathologischen Prozesse im Lungenparenchym begnügen, so machen die Zweifel stutzig, welche im Kreise der Pathologen über die ersten Zeichen tuberkulöser Gewebsreaktion herrschen. Ein Teil der Autoren ${ }^{1}$ spricht nach Baumgartens Vorgang als frühestes Merkmal der tuberzulösen Infektion das Auftreten zahlreicher Mitosen in fixen Zellen an, ein anderer dagegen, im Sinne Metschnikoffs, die Häufung beweglicher, aus dem Blute stammender Zellen mit phagozytären Eigenschaften, ein dritter - Weigert und seine Schüler - die Läsion der elastischen Gewebselemente. Selbst wenn man sich aber über diese Streitfragen eine bestimmte Ansicht gebildet Bd. XXIX.

1 Vgl. die ausfübrliche Literaturübersicht bei Wechsberg. Zieglers Beiträge. 
hat, so bleibt die Verwertung der vorgefundenen initialen Lungenveränderung für die Frage der primären Ansiedlung der Bazillen noch immer zweifelhaft. Namentlich Orth ${ }^{1}$ hat betont, daB auf Grund der histologischen Veränderungen im Lungenparenchym eine sichere Entscheidung über die bronchogene oder metastatische Entstehung der pathologischen Prozesse nicht angängig sei. Dieses Bedenken gegen die ätiologische Bedeutung der histologischen Untersuchungsergebnisse ist so schwerwiegend, $d a B$ es uns durchaus wieder auf den ersten Weg, den direkten Nachweis der ersten Etablierung der Bazillen in Inhalationsexperimenten, zurückdrängt. Bei diesen Versuchen liegt freilich eine Hauptschwierigkeit in der richtigen Dosierung. Diese Schwierigkeit durfte ich indes zu überwinden hoffen. Mit Hilfe der im hiesigen Institut ausgebildeten Technik, welche die Inhalationsdosis beliebig zu variieren gestattet, mußte es möglich sein, Material zu gewinnen, in welchem trotz relativ geringer Bazillenzahl deren Nachweis vielleicht nicht allzu schwierig sein würde.

Die Versuchsanordnung war die gleiche wie bei den biologischen Untersuchungen: Gleichzeitig mit den Meerschweinchen, welche für die letzteren verwendet warden, lieB ich Tiere mittleren Alters serienweise je 50000,100000 und 1000000 Bazillen inhalieren und tötete sie - zumeist durch Nackensehlag - nach 1 bis $2,12,24,3 \times 24,6 \times 24$ Stunden und später. Unverzüglich danach lieB ich in die ohne Eröffnung des Thorax freigelegte, kopfwärts abgebundene Trachea durch eine vorsichtig eingestochene Kanüle ca $20 \mathrm{~cm} 10$ prozentiges Formalin unter ganz geringem Druck einlaufen, wartete etwa 2 Stunden und nahm hierauf die Brustorgane in toto heraus. Die Lungen sind alsdann in natürlicher Lage fixiert und können nun in beliebiger Weise weiterverarbeitet werden. Einzelne Lungenlappen wurden sorgfältig von der Lungenwurzel abgeschnitten, in Alkobol steigender Konzentration gehärtet, im ganzen oder geteilt in Paraffin eingebettet und in Serienschnitte von $10 \mu$ Dicke zerlegt, welche auf dem Objektträger vermittelst 30 prozentigen erwärmten Alkohols und nachfolgenden Einlegens in den Brutschrank von $37^{\circ}$ für mehrere Stunden fixiert wurden. Ihre Färbung erfolgte zu allgemeiner Orientierung mit Hämatoxylin und Eosin, zum Nachweis der Bazillen in der üblichen Weise mit Karbolfuchsin (1 Stunde ohne Erwärmen, dann salzsaurer Alkohol 1 bis 2 Sekunden, 60 proz. Alkohol bis zur Mattrosafärbung) und Methylenblau. Auch kompliziertere, auf das Studium bestimmter Gewebsbestandteile, wie der Mitosen und elastischen Fasern,

\footnotetext{
1 Orth, Diskussionsbemerkung za Westenhöffers Vortrag. Berliner klin. Wochenschrift. 1904. Nr. 8. S. 203.
} 
gerichtete Färbemethoden habe ich angewandt, werde jedoch über sie und über die mit ihnen gewonnenen Ergebnisse in einer späteren Arbeit berichten und mich in dieser. Mitteilung wesentlich auf die Befunde beschränken, die für die Frage der primären Bazillenansiedlung bedeutsam sind.

Während ich in den Schnittserien der Tiere mit einer Inhalationsdosis von 50000 und 100000 Bazillen in frühen Stadien trotz deutiicher Gewebsveränderungen fast stets vergeblich nach Bazillen suchte, fanden sich in den mit 1 Million Bazillen infizierten Lungen in mehreren Serien Tuberkelbazillen in genügender Anzahl und in so frühem Stadium, daB ich über die ersten Vorgänge nach der Inhalation der keimhaltigen Spraytröpfchen eine sichere Orientierung gewinnen konnte. - In der Lunge eines 2 Stunden nach der Inhalation getöteten Tieres fand ich zunächst im Epithelbelag der kleineren und kleinsten Bronchien hier und da Zellen, welche ohne nachweisbare Schädjgung Tuberkelbazillen, manchmal in erheblicher Anzahl (10 bis 15), enthielten. Zumeist waren es pigmenthaltige, nur selten pigmentfreie Zellen, welche die Bazillen beherbergten. Gelegentlich erhob sich eine tuberkelbazillenhaltige Zelle, namentlich wenn sie mit reichlichem Pigment und zablreichen Bazillen erfüllt war, etwas über das Niveau der übrigen hervor, andere ragten weit darüber hinaus, andere hingen nur noch mit einer schmalen Brücke mit dem Epithelsaum zusammen, andere endlich waren gänzlich von ihm abgelöst und lagen flach auf dem Flimmerbesatz der noch haftenden Zylinderzellen. Manchmal hatten sich mehrere Zellen im Zusammenhang vōllig abgelöst und so eine gröBere Epithellücke, zurückgelassen. Solche freie Zellen enthielten fast durchgehends auBerordentlich zahlreiche Bazillen und sehr viel Pigment. Bei vielen von ihnen ist nach Gestalt, Struktur und Lagerung die Herkunft rom Bronchialepithel zweifellos. Manche von ihnen haben eine ausgesprochen flache Form und dürften dem respiratorischen Plattenepithel der tiefer gelegenen Luftwege zuzurechnen sein. Nur selten habe ich tuberkelbazillenhaltige Zellen beobachtet, deren kleinere, rundlichere Gestalt und gelappter, dunkel gefärbter Kern auf ibre Leukozytennatur schließen lieB; Staub war in ihnen sehr selten vorhanden. Besonders zablreiche tuberkelbazillenhaltige Zellen fanden sich in einer Schnittserie, welche gerade in günstiger Weise durch die Teilungsstelle eines etwas gröBeren Bronchus in seine feineren Äste verlief, an der inneren Scheitelpartie der Gabel. An solchen Stellen habe ieh auch subepithelial gelegene, bazillenhaltige Zellen - meist pigmenthaltige, epitheloide - in geringer Zahl angetroffen. Ganz vereinzelt fanden sich endlich noch Tuberkelbazillen, die anscheinend frei auf oder zwischen den Zellen lagen, einige Male gleichfalls bereits unterhalb der Epitheldecke. 
Analoge, nur quantitativ stetig abnehmende Befunde bieten die weiteren Luftwege bis zu den Alveolen hin. Während sich in den Bronchioli respiratorii noch relativ zahlreiche Tuberkelbazillen finden, werden sie in dem Ductuli alveolares und den Alveolen selbst spärlich und liegen nur zu 1 bis 2, selten einmal bis 4 in einer Zelle. Fast stets handelt es sich hier um pigmenthaltige, platte Zellen, welche meist im Niveau der Epitheldecke liegen und ganz unzweifelhaft in das Zellmosaik derselben hineingehören. Manche sind mehr oder weniger von ihren Nachbarn losgelöst und liegen dann, gelegentlich anscheinend frei, im Lumen der Alveole. Ofters sind mehrere Epithelien derselben Alveole abgehoben und erfüllen ihren Hohlraum; in solchen Fällen gesellen sich zu ihnen manchmal noch einige rote Blutkörperchen. Höchst selten babe ich Bazillen gefunden, welche einer Epithelzelle offenbar nur auflagen; es ist vielleicht nicht ohne Bedeutung, daß dies stets pigmentfreie Zellen wareu. Einmal sah ich eine pigmenthaltige Epithelzelle, welche den Bacillus nur zur Hälfte beherbergte, während der Rest noch frei ins Lumen der Alveole hineinragte. In seltenen Füllen fand sich eine als Leukozyt sicher erkennbare, bazillenhaltige Zelle zwischen den Epithelien oder im Innern der Alveole, einmal in dichtester Nähe eines KapillargefäBchens und nur durch dessen einschichtige Endothelwand von seinem Lumen getrennt. Im interalveolären Gewebe sind, ähnlich wie an den Brouchien und Bronchiolen, vereinzelte bazillenhaltige epitheloide und leukozytäre Elemente sowie anscheinend freie Bazillen anzutreffen.

Diese Bilder stimmen gut überein mit den von Morel und Dalous', Herxheimer ${ }^{2}$ sowie Watanabe ${ }^{3}$ erhobenen Befunden nach intratrachealer Injektion von Tuberkelbazillenemulsionen an denjenigen Stellen, an welche relativ mäBige Mengen von Bazillen hingelangt waren. Sie sind nicht anders zu deuten, als daB die inhalierten Tuberkelbazillen hauptsächlich direkt an die Epithelzellen herantreten, von ibnen aufgenommen werden und in ihnen entweder ins Lumen der Lufträume oder ins tiefere Gewebe gelangen. Daneben findet eine Aufnahme von Bazillen durch Leukozyten und eine anscheinend freie Einlagerung von Bazillen im Gewebe statt. Utber das fernere Schicksal der Bazillen werden weitere, bereits im Gange befindliche Untersuchungen Aufschluß geben. Nach den geschilderten Befunden muß die Möglichkeit zur Tuberkelbildung innerhalb der Luftwege, in den Alveolen und Bronchien ebensogut wie in den interstitiellen Gewebspartien und ihre Weiterverschleppung aus

1 Morel et Dalous, Arch. de méd. expér. 1901.

2 Herxheimer, Zieglers Beilräge. Bd. XXXII.

${ }^{3}$ Watanabe, Ebenda. Bd. XXXI. 
Aufnahme inhalierter Tuberikelbazillen in die Lunge. 507

diesen auf dem Lymphwege zugegeben werden. Welcher von diesen Wegen der auch unter natürlichen Verhältnissen beschrittenste ist, und ob er vielleicht nach Tierart, Alter, Infektionsart usw. stark wechselt, das müssen besondere Untersuchungen lehren. Hier kam es mir zunächst nur darauf an, durch den direkten mikroskopischen Nachweis der inhalierten Bazillen in den Alveòlen und feinsten Bronchien einen ganz zweifellosen Beweis dafür zu erbringen, daß die Inhalation die beigemengten Tuberkelbazillen unmittelbar an die Stätte schafft, an der bzw. von der aus die Wucherung der Bazillen und die Entwicklung des Tuberkels beginnen kann.

Es ist demnach mit völliger Eindeutigkeit erwiesen, daB inhalierte Tuberkelbazillen mit Leichtigkeit auf direktem Wege in die Lungen gelangen. Dieses Resultat steht durchaus im Einklang mit den Ergebnissen älterer und neuerer im hiesigen hygienischen Institut gemachten Beobachtungen über die sichere und schnelle Erzeugung einer ausgedehnten Lungentuberkulose durch Inhalation versprayter Aufschwemmungen ron Tuberkelbazillenkultur oder phthisischem Sputum. Von ganz besonderer Beweiskraft ist unter diesen Beobachtungen die Erzeugung von Lungentuberkulose an tracheotomierten Tieren, bei denen jeder andere Infektionsweg ausgeschlossen war, in der kürzesten Frist, die überhaupt bei Tuberkuloseexperimenten vorkommt (Findel). ${ }^{1}$ Bei keinem anderen Infektionsmodus geht die Entwicklung der Lungentuberkulose mit solcher Schnelligkeit vor sich, wie nach der Inhalation mäßiger Mengen von tuberkelbazillenhaltigen Tröpf́chen oder Stäubchen. Sind doch selbst bei den kleinsten Dosen inhalierter Tuberkelbazillen schon nach 20 Tagen wohlausgebildete Tuberkel in den Lungen zu konstatieren, während nach Verfütterung und stomachaler Einverleibung vergleichsweise enormer Dosen mindestens die doppelte Zeit verfließt, ehe Lungentuberkel ausgebildet sind.

So strömen von allen Seiten die Beweise zusammen, um die Tatsache zu sichern, daß für inhalierte Tuberkelbazillen der unmittelbare Eintritt in die Lungen leicht ist, und wenn uns auch hier, wie stets, die Übertragung der an Versuchstieren gewonnenen Ergebnisse auf den unter natürlichen Bedingungen lebenden Menschen vorsichtige Zurückhaltung auferlegt, so ist doch nicht mehr daran zu zweifeln, daB auch für den Menschen die Inhalation einen besonders leicht gangbaren Infektionsweg repräsentiert.

${ }^{1}$ Findel, Vergleichende Untersachungen über Inhalations- and Fütterungstuberkulose. Diese Zeitschrift. 1907. Bd. LVII. 